\title{
Possible association between moderate intellectual disability and weight gain in valproic acid-treated patients with epilepsy
}

This article was published in the following Dove Press journal:

Neuropsychiatric Disease and Treatment

7 April 2015

Number of times this article has been viewed

\author{
Yukiko Tanamachi' \\ Junji Saruwatari' \\ Madoka Noai' \\ Ryoko Kamihashi' \\ Hiromi Soraoka' \\ Yuki Yoshimori' \\ Naoki Ogusu' \\ Kentaro Oniki' \\ Norio Yasui-Furukori ${ }^{2}$ \\ Takateru Ishitsu ${ }^{3,4}$ \\ Kazuko Nakagawa ${ }^{1,5}$ \\ 'Division of Pharmacology and \\ Therapeutics, Graduate School of \\ Pharmaceutical Sciences, Kumamoto \\ University, Kumamoto, Japan; \\ ${ }^{2}$ Department of Neuropsychiatry, \\ Hirosaki University School of \\ Medicine, Hirosaki, Japan; ${ }^{3}$ Kumamoto \\ Saishunso National Hospital, Koshi, \\ Japan; ${ }^{4}$ Kumamoto Ezuko Ryoiku Iryo \\ Center, Kumamoto, Japan; ${ }^{5}$ Center \\ for Clinical Pharmaceutical Sciences, \\ Kumamoto University, Kumamoto, \\ Japan
}

Background: Although patients with moderate intellectual disability (ID) are known to have higher rates of being overweight and obese than those without ID, there are no current data regarding the relationship between ID and weight gain in epilepsy patients treated with valproic acid (VPA).

Patients and methods: The possible association between moderate ID and an overweight status at the time of initiation of VPA therapy (baseline) was investigated using a logistic regression analysis in 143 patients with epilepsy. Among the 119 nonoverweight patients at baseline, the longitudinal association between moderate ID and the weight status during VPA therapy was retrospectively examined using a Cox hazards regression analysis and the generalized estimating equations approach, while also paying careful attention to associations with other patient characteristics.

Results: The proportion of patients with moderate ID was $52.4 \%$ among the 143 study subjects. The presence of moderate ID was not associated with an overweight status at baseline $(P=0.762)$. Among the nonoverweight patients at baseline, 16 subjects were newly diagnosed as being overweight during treatment with VPA (3.6 \pm 2.1 years). The presence of moderate ID was significantly associated with the incidence of an overweight status after starting VPA therapy (adjusted hazard ratio $=6.72, P=0.007$ ). The patient age at baseline and treatment with co-administered carbamazepine, clobazam, and zonisamide significantly influenced the degree of weight fluctuation during VPA therapy among the patients with moderate ID $(P<0.001$, $P<0.001, P=0.002$, and $P=0.028$, respectively), whereas only patient age at baseline affected this parameter among the patients without moderate ID $(P=0.022)$.

Conclusion: The present findings suggest that the weight status should be carefully monitored in VPA-treated patients with moderate ID, especially those receiving other co-administered antiepileptic drugs that facilitate weight gain, such as carbamazepine.

Keywords: overweight, weight status, obesity, antiepileptic drug, longitudinal analysis

\section{Introduction}

People with intellectual disability (ID) experience significant health inequalities compared with the general population. ${ }^{1,2}$ For example, patients with ID have higher mortality rates, a shorter life expectancy, and higher levels of unmet health needs. Previous studies have indicated that individuals with ID have higher levels of an overweight status and obesity than those without ID, ${ }^{1-5}$ and moderate, but not severe, ID has been reported to be associated with being overweight. ${ }^{3,4}$

It has also been reported that the risk of developing ID is high among patients with epilepsy. ${ }^{6-9}$ Valproic acid (VPA) is one of the most widely prescribed antiepileptic drugs worldwide. ${ }^{10}$ Long-term treatment with VPA has been shown to be associated 
with metabolic and endocrine disorders, such as weight gain and hyperinsulinemia, which may contribute to the increased cardiovascular risks observed in patients with epilepsy. ${ }^{11-13}$ Although the real incidence and magnitude of VPA-induced weight gain is unknown, the reported frequency of weight gain varies between $10 \%$ and $70 \% .^{11,12}$ Several potential risk factors for VPA-induced weight gain have been identified, including puberty and a pretreatment overweight status. ${ }^{11,12}$ However, there are presently no data available regarding the relationship between ID and VPA-induced weight gain.

The primary objective of this exploratory study was to retrospectively investigate the influence of moderate ID on an overweight status in VPA-treated patients with epilepsy. Furthermore, we determined potentially specific factors affecting weight gain in VPA-treated patients with moderate ID.

\section{Materials and methods}

A clinic-based retrospective longitudinal analysis was conducted among 143 Japanese patients (89 males and 54 females) with epilepsy treated with VPA at Kumamoto Saishunso National Hospital in Kumamoto, Japan, between June 1989 and April 2011. Demographic and clinical data were obtained retrospectively from the subjects' medical records. The study protocol was approved by the ethics committees of Kumamoto Saishunso National Hospital and the Faculty of Life Sciences, Kumamoto University (Kumamoto, Japan). Informed consent, including a statement regarding the privacy policy, was obtained in writing from each patient and his/her parent before entry into the study.

Patients were included if they fulfilled all the following conditions: younger than 18 years of age at the start of VPA therapy (baseline); treatment with sustained-release VPA for 6 months or longer and not taking any drugs that may alter the body weight, except for antiepileptic drugs; and no history of severe or profound ID, eg, severe motor and intellectual disabilities, as well as other psychiatric disorders. The type of seizure and epileptic syndrome was classified according to the guidelines of the International League Against Epilepsy. ${ }^{14}$ In all patients, VPA therapy was chosen according to the treatment guidelines of the Japan Epilepsy Society. The dose of VPA was escalated at weekly intervals by $5-10 \mathrm{mg} / \mathrm{kg} /$ day for each step up to the maximum tolerated dose. Clinical data were recorded at each follow-up visit. For each patient, the data included the patient's weight, dose, and schedule of VPA and concomitant medications, evaluated at all visits during treatment with VPA. We used the International Classification of Diseases, 10th Revision (ICD-10) to identify individuals with moderate ID (ICD-10 F71, consistent with an IQ score of 35-49) and defined patients with an IQ score of $\geq 50$ as being without moderate ID.

The height and weight of each subject were measured, and the body mass index (BMI) was calculated. Since the BMI in childhood changes substantially with age, ${ }^{15}$ the age- and sex-specific international BMI cutoff value for an overweight status in children, which was established by Cole et $\mathrm{al}^{15}$ was used for diagnosis of being overweight when the patient was 17 years of age or under. When the patient was 18 years of age or older during VPA therapy, an overweight status was diagnosed as a $\mathrm{BMI} \geq 25 \mathrm{~kg} / \mathrm{m}^{2}$. We determined the BMI gap in each patient at every follow-up visit using the following equation: BMI gap = patient's BMI value - cutoff value for overweight.

Categorical and continuous variables were compared using Fisher's exact test and the Mann-Whitney $U$-test, respectively. At baseline, the strength of the association between moderate ID and the risk of being overweight was measured as the odds ratio using a logistic regression analysis in all VPA-treated patients with epilepsy. Among the patients who were not overweight at baseline, the cumulative incidence of becoming overweight according to the presence or absence of moderate ID was plotted based on the Kaplan-Meier method, and the multivariable-adjusted hazard ratio (HR) was determined using a Cox proportional hazards model. The odds ratio and HR were adjusted for potentially confounding factors, ${ }^{11,16}$ ie, the patient age at baseline, sex, dose of VPA, seizure locus, and the co-administration of antiepileptic drugs that may influence the body weight (ie, carbamazepine, clobazam, gabapentin, zonisamide) and other antiepileptic drugs. In order to identify specific factors influencing the fluctuation in weight in the patients with moderate ID, the associations between the patient age at baseline, sex, dose of VPA, seizure locus and coadministration of antiepileptic drugs and the longitudinal changes in the BMI gap were also measured using adjusted partial regression coefficients $(B)$ separately in the patients with and without moderate ID. The $B$-values were calculated employing a multiple linear regression model based on the generalized estimating equations approach. In the multiple linear regression model, the variance structure model was best described using an autoregressive model. In order to assess the cumulative incidence of an overweight status and the multivariable-adjusted HR obtained using the Cox proportional hazards model, we included all data acquired at every follow-up visit during the VPA therapy period. In order to assess the longitudinal changes in the BMI gap using the generalized estimating equations approach, we extracted 
the values for the BMI gap at yearly intervals after starting the VPA therapy. A $P$-value of $<0.05$ was considered to be statistically significant. All statistical analyses were performed using the SPSS software package for Windows (version 17.0, IBM Japan Ltd., Tokyo, Japan).

\section{Results}

The prevalence of moderate ID was 52.4\% among all 143 VPA-treated patients with epilepsy. The patient characteristics at baseline according to the presence or absence of moderate ID are shown in Table 1. The frequency of being overweight at baseline was $18.7 \%$ and $14.7 \%$ in the patients with and without moderate ID, respectively $(P>0.05)$. Significant differences were observed between the patients with moderate ID and those without moderate ID in terms of the daily VPA dose; number of patients receiving
VPA only; number of patients treated with co-administered carbamazepine, clobazam, or phenytoin; seizure locus; and seizure type (Table 1). According to the logistic regression analysis in the 143 patients, moderate ID was not associated with the incidence of an overweight status at baseline (odds ratio $=1.18,95 \%$ confidence intervals $=0.40-3.53$, $P=0.762$ ).

The patient characteristics of the 119 patients who were not overweight at baseline according to the presence or absence of moderate ID are shown in Table 1. Significant differences were also observed between the patients with moderate ID and those without moderate ID in the daily VPA dose; number of patients receiving VPA only; number of patients treated with co-administered carbamazepine, clobazam or phenytoin; seizure locus; and seizure type (Table 1). No patients were treated with gabapentin, which may

Table I Demographic and clinical characteristics of the study subjects

\begin{tabular}{|c|c|c|c|c|}
\hline \multirow[t]{2}{*}{ Characteristics } & \multicolumn{2}{|c|}{$\begin{array}{l}\text { All patients included at baseline } \\
(n=143)\end{array}$} & \multicolumn{2}{|c|}{$\begin{array}{l}\text { Patients who were not overweight } \\
\text { at baseline }(n=\mid 19)\end{array}$} \\
\hline & $\begin{array}{l}\text { Patients with } \\
\text { moderate ID }(n=75)\end{array}$ & $\begin{array}{l}\text { Patients without } \\
\text { moderate ID }(n=68)\end{array}$ & $\begin{array}{l}\text { Patients with } \\
\text { moderate ID }(n=6 I)\end{array}$ & $\begin{array}{l}\text { Patients without } \\
\text { moderate ID }(n=58)\end{array}$ \\
\hline Age (years) & $10.8 \pm 4.4[2.0-17.8]$ & $10.8 \pm 4.1[3.4-17.9]$ & $11.0 \pm 4.4[2.0-17.8]$ & $10.7 \pm 4.1[3.6-17.9]$ \\
\hline Male/female & $52(69.3 \%) / 23(30.7 \%)$ & $37(54.4 \%) / 3$ I (45.6\%) & $43(70.5 \%) / 18(29.5 \%)$ & $31(53.4 \%) / 27(46.6 \%)$ \\
\hline Body weight (kg) & $36.1 \pm 16.6[8.9-82.0]$ & $37.2 \pm 16.8[13.0-85.0]$ & $34.4 \pm \mid 4.6[8.9-72.0]$ & $34.7 \pm 15.0[13.0-65.0]$ \\
\hline Height (cm) & $|36.| \pm 24.4[77.7-|76|]$. & $138.5 \pm 23.2[88.7-\mid 74.3]$ & $|37.| \pm 23.7[77.7-\mid 76.0]$ & $138.0 \pm 23.2[94.9-174.3]$ \\
\hline BMI $\left(\mathrm{kg} / \mathrm{m}^{2}\right)$ & $18.4 \pm 3.3[13.6-28.7]$ & $18.3 \pm 3.6[12.8-29.9]$ & $17.3 \pm 2.1[13.6-23.3]$ & $17.2 \pm 2.3[12.8-22.4]$ \\
\hline BMI gap $\left(\mathrm{kg} / \mathrm{m}^{2}\right)$ & $-2.4 \pm 3.3[-8.2$ to 7.2$]$ & $-2.5 \pm 3.1[-7.5$ to 9.1$]$ & $-3.6 \pm 2.2[-8.2$ to 0.0$]$ & $-3.5 \pm 1.5[-7.5$ to -0.3$]$ \\
\hline Overweight & $14(18.7 \%)$ & $10(14.7 \%)$ & $0(0 \%)$ & $0(0 \%)$ \\
\hline Daily VPA dose (mg/day) & $792.3 \pm 534.6[200-2,500]^{\mathrm{a}}$ & $60 I .4 \pm 373.2[200-2,000]^{\mathrm{a}}$ & $744.6 \pm 490.0[200-2,400]^{\mathrm{a}}$ & $553.4 \pm 315.9[200-1,600]^{\mathrm{a}}$ \\
\hline $\begin{array}{l}\text { Patients who had been treated } \\
\text { with VPA only }\end{array}$ & $28(37.3 \%)^{\mathrm{a}}$ & $46(67.6 \%)^{\mathrm{a}}$ & $23(37.7 \%)^{\mathrm{a}}$ & $41(70.7 \%)^{\mathrm{a}}$ \\
\hline $\begin{array}{l}\text { Patients who had been treated } \\
\text { with VPA and other antiepileptic drug(s) }\end{array}$ & $47(62.7 \%)^{\mathrm{a}}$ & $22(32.4 \%)^{\mathrm{a}}$ & $38(62.3 \%)^{\mathrm{a}}$ & $17(29.3 \%)^{\mathrm{a}}$ \\
\hline \multicolumn{5}{|l|}{ Co-administration } \\
\hline $\mathrm{CBZ}$ & $25(33.3 \%)^{\mathrm{a}}$ & $9(13.2 \%)^{\mathrm{a}}$ & $18(29.5 \%)^{\mathrm{a}}$ & $5(8.6 \%)^{a}$ \\
\hline CLB & $9(12.0 \%)^{a}$ & $\mathrm{I}(\mathrm{I} .5 \%)^{\mathrm{a}}$ & $8(13.1 \%)^{a}$ & $0(0 \%)^{\mathrm{a}}$ \\
\hline CZP & $6(8.0 \%)$ & $3(4.4 \%)$ & $4(6.6 \%)$ & 3 (5.2\%) \\
\hline ETS & $3(4.0 \%)$ & $4(5.9 \%)$ & $3(4.9 \%)$ & $4(6.9 \%)$ \\
\hline PB & $5(6.7 \%)$ & $2(2.9 \%)$ & $3(4.9 \%)$ & $2(3.4 \%)$ \\
\hline PHT & $6(8.0 \%)^{a}$ & $0(0 \%)^{\mathrm{a}}$ & $6(9.8 \%)^{\mathrm{a}}$ & $0(0 \%)^{\mathrm{a}}$ \\
\hline TPM & I (I.3\%) & $0(0 \%)$ & I (1.6\%) & $0(0 \%)$ \\
\hline ZNS & $12(16.0 \%)$ & $4(5.9 \%)$ & II (I8\%) & $4(6.9 \%)$ \\
\hline \multicolumn{5}{|l|}{ Seizure locus } \\
\hline Generalized & $28(37.3 \%)^{\mathrm{a}}$ & $43(63.2 \%)^{\mathrm{a}}$ & $22(36.1 \%)^{a}$ & $36(62.1 \%)^{\mathrm{a}}$ \\
\hline Partial & $47(62.7 \%)^{\mathrm{a}}$ & $25(36.8 \%)^{\mathrm{a}}$ & $39(63.9 \%)^{a}$ & $22(37.9 \%)^{\mathrm{a}}$ \\
\hline \multicolumn{5}{|l|}{ Seizure type } \\
\hline Idiopathic & $\mathrm{I}(\mathrm{I} .3 \%)^{\mathrm{a}}$ & $4 \mid(60.3 \%)^{a}$ & $\mathrm{I}(\mathrm{I} .6 \%)^{\mathrm{a}}$ & $37(63.8 \%)^{\mathrm{a}}$ \\
\hline Symptomatic & $22(29.3 \%)^{\mathrm{a}}$ & $8(11.8 \%)^{\mathrm{a}}$ & $19(31.3 \%)^{a}$ & $8(13.8 \%)^{a}$ \\
\hline Cryptogenic & $52(69.3 \%)^{\mathrm{a}}$ & $19(27.9 \%)^{\mathrm{a}}$ & $4 \mathrm{I}(67.2 \%)^{\mathrm{a}}$ & $13(22.4 \%)^{\mathrm{a}}$ \\
\hline
\end{tabular}

Notes: Data were expressed as the means \pm standard deviations [range] or number (\%). The BMI gap in each patient using the following equation: BMI gap $=$ patient's BMI

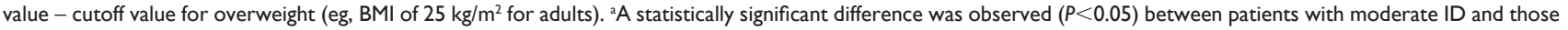
without via Fisher's exact test or the Mann-Whitney U-test.

Abbreviations: ID, intellectual disability; BMI, body mass index; VPA, valproic acid; CBZ, carbamazepine; CLB, clobazam; CZP, clonazepam; ETS, ethosuximide; PB, phenobarbital; PHT, phenytoin; TPM, topiramate; ZNS, zonisamide. 


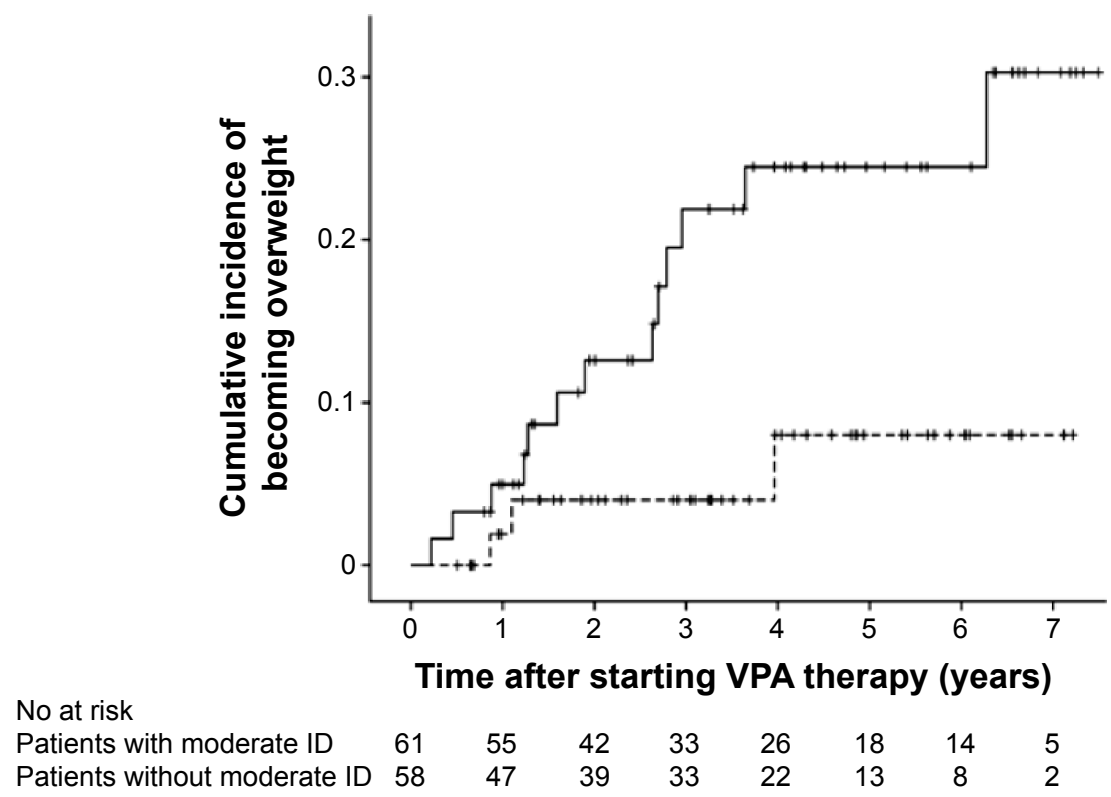

Figure I Kaplan-Meier estimates of the cumulative incidence of becoming overweight in the VPA-treated patients with epilepsy. Note: The solid and dotted lines represent patients with moderate ID $(n=6 I)$ and those without moderate ID ( $n=58)$, respectively. Abbreviations: VPA, valproic acid; ID, intellectual disability.

influence the body weight, ${ }^{16}$ during VPA therapy. The mean duration of follow-up was $3.6 \pm 2.1$ years $(4.1 \pm 2.1$ years for the patients with moderate ID; $3.1 \pm 1.9$ years for those without moderate ID). Sixteen (13.4\%) patients (13 [21.3\%] patients with moderate ID, 3 patients [5.2\%] without moderate ID] were newly diagnosed to be overweight during the VPA therapy period. Figure 1 shows the cumulative incidence of becoming overweight in the patients with and without moderate ID. The presence of moderate ID was significantly associated with a higher incidence of an overweight status in the Cox regression model (HR $=6.72,95 \%$ confidential intervals $=1.66-27.15, P=0.007)$, which was adjusted for potential confounding factors, ie, the patient age at baseline, sex, dose of VPA, seizure locus, and co-administration of antiepileptic drugs that can influence the body weight (carbamazepine, clobazam, zonisamide) and other antiepileptic drugs.

In order to identify factors influencing longitudinal changes in body weight during VPA therapy, we investigated the associations between the patient age at baseline, sex, dose of VPA, seizure locus, and co-administration of antiepileptic drugs and the longitudinal changes in the BMI gap separately in the patients with and without moderate ID (Table 2). Among the patients with moderate ID, the co-administration of carbamazepine and clobazam was significantly associated with an increased BMI gap, while a younger age at baseline and the co-administration of zonisamide was associated with a decreased BMI gap (Table 2). Among the patients without moderate ID, a younger age at baseline was associated with a decreased BMI gap, whereas none of the other patient characteristics were associated with the longitudinal changes in the BMI gap (Table 2). Table 3 shows the number of patients treated with carbamazepine, clobazam, or zonisamide at each time point used in the evaluation of the longitudinal changes in the BMI gap during VPA therapy. The number of patients treated with carbamazepine and clobazam tended to be higher among the subjects with moderate ID than among those without (Table 3 ). The daily doses of carbamazepine and clobazam during VPA therapy also tended to be greater in the patients with moderate ID than in those without moderate ID (Figure 2), whereas there were no differences in the dose of zonisamide (data not shown).

\section{Discussion}

The findings of this retrospective longitudinal study showed that moderate ID is associated with weight gain in patients with epilepsy under VPA therapy. It is known that VPAinduced weight gain may be associated with pathological consequences related to obesity, such as dyslipidemia, diabetes mellitus, and atherosclerosis. ${ }^{11,12,17}$ Therefore, identifying patients at risk for weight gain will help clinicians to select the appropriate treatment and appropriately counsel these patients to avoid weight gain. ${ }^{11}$ The results of previous clinical studies suggest that various risk factors contribute to VPA-induced weight gain, including a female sex, puberty, a long duration of therapy, generalized seizures, and a pretreatment overweight status. ${ }^{11,12}$ The findings of the current study 
Table 2 Factors associated with the longitudinal changes in the BMI gap during VPA therapy identified in the multiple linear regression analysis using a generalized estimating equations approach

\begin{tabular}{llll}
\hline Covariate & B & SE & P-value \\
\hline Patients with moderate ID & & & \\
Age at baseline & -0.3 I & 0.06 & $<0.00$ I \\
Female sex & 0.34 & 0.68 & 0.621 \\
Generalized seizure & 0.43 & 0.49 & 0.379 \\
Daily VPA dose & 0.00 & 0.00 & 0.758 \\
Co-administration of CBZ & 1.08 & 0.28 & $<0.00$ I \\
Co-administration of CLB & 1.01 & 0.33 & 0.002 \\
Co-administration of ZNS & -0.95 & 0.43 & 0.028 \\
Co-administration of other & 0.16 & 0.25 & 0.525 \\
antiepileptic drugs & & & \\
Patients without moderate ID & & & \\
Age at baseline & -0.11 & 0.05 & 0.022 \\
Female sex & 0.32 & 0.37 & 0.379 \\
Generalized seizure & 0.18 & 0.37 & 0.624 \\
Daily VPA dose & 0.00 & 0.00 & 0.733 \\
Co-administration of CBZ & 0.29 & 0.29 & 0.317 \\
Co-administration of CLB & 0.14 & 0.34 & 0.648 \\
Co-administration of ZNS & -0.31 & 0.31 & 0.319 \\
Co-administration of other & 0.23 & 0.32 & 0.470 \\
antiepileptic drugs & & & \\
\hline Not The SE & &
\end{tabular}

Notes: The $B, S E$ and $P$-values were calculated by a longitudinal multiple linear regression analysis that included all covariates listed in the table based on the generalized estimating equations approach. The BMI gap in each patient using the following equation: BMI gap = patient's BMI value - cutoff value for overweight (eg, BMI of $25 \mathrm{~kg} / \mathrm{m}^{2}$ for adults).

Abbreviations: ID, intellectual disability; BMI, body mass index; VPA, valproic acid; $B$, adjusted partial regression coefficient; SE, standard error; $C B Z$, carbamazepine; CLB, clobazam; ZNS, zonisamide.

suggest that moderate ID may be a potential novel risk factor for weight gain in VPA-treated patients with epilepsy.

The mechanism underlying VPA-induced weight gain is a matter of debate, and various hypotheses have been proposed to explain the effects of VPA on increases in weight. Such mechanisms include dysregulation of the hypothalamic system and the effects of insulin resistance. ${ }^{11,12}$ It is known that treatment with VPA disrupts the hypothalamo-hypophysealgonadal axis in female rats, ${ }^{18}$ and it has been reported that VPA-treated patients who report weight gain develop an increased appetite and frequently consume calorie-rich beverages. ${ }^{11,12,19}$ It has also been demonstrated that people with ID, especially those with moderate ID, have higher levels of being overweight and obese than those without. ${ }^{2-5}$ Proposed reasons for this observation include high levels of inactivity and comorbid health conditions, physical disabilities, or excessive eating in individuals with ID. ${ }^{1,2,5}$ Therefore, we speculate that the more pronounced VPA-induced weight gain observed in patients with moderate ID may be attributed to ID-related physical inactivity or excessive eating, although the underlying mechanism remains unknown.
In the present study, the co-administration of carbamazepine and clobazam was significantly associated with an increased BMI gap only in the patients with moderate ID (Table 2). Previous studies have reported that patients treated with carbamazepine experience weight gain in the same manner as VPA-treated patients, ${ }^{20,21}$ and the frequency of weight gain is observed to be as high as $19.1 \%$ in patients treated with clobazam. ${ }^{22}$ Patients with epilepsy and ID often receive many antiepileptic drugs at high doses, as ID has been identified to be a potential predictor of intractable epilepsy. ${ }^{23-25}$ In the present study, the number of patients treated with carbamazepine and clobazam tented to be higher among the subjects with moderate ID than those without (Tables 1 and 3), and the doses of these antiepileptic drugs were greater in the patients with moderate ID (Figure 2). When these findings are combined, the high incidence of an overweight status among the VPA-treated patients with moderate ID may also be attributed to the higher frequencies and greater doses of the co-administered carbamazepine and clobazam in these patients, although we adjusted for these potential confounding factors in the Cox proportional hazards regression analysis.

The findings of previous clinical studies have demonstrated that a decreased appetite and weight loss are frequently reported in patients treated with zonisamide. ${ }^{26,27}$ In the present study, the co-administration of zonisamide was associated with a decreased BMI gap only in the patients with moderate ID (Table 2), whereas the number and daily dose of co-administrated zonisamide during VPA therapy did not differ between the patients with moderate ID and those without (Tables 1 and 3). Therefore, the weight lossinducing effect of zonisamide may be more pronounced in patients with moderate ID than in those without moderate ID. Nevertheless, it remains to be definitively determined why co-administered zonisamide was associated with a decreased BMI gap only in the patients with moderate ID in the current study. Therefore, further investigation with a larger number of patients is required to verify the associations discussed earlier.

Puberty has been identified to be a risk factor for weight gain in VPA-treated patients with epilepsy. ${ }^{11}$ Previous studies have suggested that the mature endocrine system in adults may be necessary for the development of VPA-related obesity. ${ }^{11}$ In this study, a younger age at baseline was found to be associated with a decreased BMI gap in either the patients with or without moderate ID (Table 2). Within our study population, the age at baseline ranged from 2.0 to 17.9 years (Table 1). The findings of the present study suggest that a 
Table 3 Number of patients treated with VPA and those co-treated with carbamazepine, clobazam or zonisamide at each time point used in the evaluation of the longitudinal changes in the BMI gap during VPA therapy

\begin{tabular}{|c|c|c|c|c|c|c|c|c|c|}
\hline $\begin{array}{l}\text { Time after starting VPA therapy } \\
\text { (years) }\end{array}$ & & 0 & $\mathbf{I}$ & 2 & 3 & 4 & 5 & 6 & 7 \\
\hline Number of patients treated & Patients with moderate ID & 61 & 47 & 36 & 31 & 29 & 22 & 13 & 11 \\
\hline with VPA therapy & Patients without moderate ID & 58 & 54 & 40 & 27 & 18 & 14 & 8 & 4 \\
\hline \multirow{4}{*}{$\begin{array}{l}\text { Number of patients } \\
\text { co-treated with CBZ }\end{array}$} & Patients with moderate ID & 18 & 11 & 10 & 10 & 9 & 7 & 5 & 2 \\
\hline & & $(29.5 \%)$ & $(23.4 \%)$ & $(27.8 \%)$ & $(32.3 \%)$ & $(31.0 \%)$ & $(31.8 \%)$ & $(38.5 \%)$ & $(18.2 \%)$ \\
\hline & Patients without moderate ID & 5 & 7 & 3 & 2 & I & 0 & 2 & 0 \\
\hline & & $(8.6 \%)$ & $(13.0 \%)$ & $(7.5 \%)$ & $(7.4 \%)$ & $(5.6 \%)$ & $(0 \%)$ & $(25.0 \%)$ & $(0 \%)$ \\
\hline \multirow{4}{*}{$\begin{array}{l}\text { Number of patients } \\
\text { co-treated with CLB }\end{array}$} & Patients with moderate ID & 8 & 5 & 6 & 5 & 6 & 5 & 4 & 5 \\
\hline & & $(13.1 \%)$ & $(10.6 \%)$ & $(16.7 \%)$ & $(16.1 \%)$ & $(20.7 \%)$ & $(22.7 \%)$ & $(30.8 \%)$ & $(45.5 \%)$ \\
\hline & Patients without moderate ID & 0 & 2 & i & 4 & 2 & 0 & 2 & i \\
\hline & & $(0 \%)$ & $(3.7 \%)$ & $(2.5 \%)$ & $(14.8 \%)$ & $(11.1 \%)$ & $(0 \%)$ & $(25.0 \%)$ & $(25.0 \%)$ \\
\hline \multirow[t]{4}{*}{ Number of patients co-treated with ZNS } & Patients with moderate ID & II & 2 & 3 & 2 & 2 & I & 0 & 0 \\
\hline & & $(18.0 \%)$ & $(4.3 \%)$ & $(8.3 \%)$ & $(6.5 \%)$ & $(6.9 \%)$ & $(4.5 \%)$ & $(0 \%)$ & $(0 \%)$ \\
\hline & Patients without moderate ID & 4 & 3 & 2 & I & I & I & 0 & 0 \\
\hline & & $(6.9 \%)$ & $(5.6 \%)$ & $(5.0 \%)$ & (3.7\%) & $(5.6 \%)$ & (7.1\%) & $(0 \%)$ & $(0 \%)$ \\
\hline
\end{tabular}

Note: Data were expressed as the number (\%).

Abbreviations: ID, intellectual disability; BMI, body mass index; VPA, valproic acid; CBZ, carbamazepine; CLB, clobazam; ZNS, zonisamide.

younger age at baseline may be associated with a low risk of VPA-induced weight gain in children, regardless of the presence of moderate ID.

A major limitation of this study is the study design, which involved a retrospective investigation of a small number of patients. Additionally, since we included only VPA-treated patients, we were unable to determine the relationships between the presence of moderate ID and the weight status in the patients treated with other antiepileptic drugs and nonepileptic subjects. Furthermore, several potential covariates associated with being overweight were not included, such as a pretreatment overweight status, dietary habits, physical activity level, presence of sleep apnea, blood concentrations of VPA and other antiepileptic drugs, blood ammonia level, electroencephalogram finding, and type(s) of disability. Sleep apnea, especially, has been reported to be associated with weight gain as well as cognitive function..$^{28,29}$ However, since we did not screen sleep apnea in the study subjects, we could not identify the patients with sleep apnea among the subjects included. Finally, since we excluded patients with a history of severe or profound ID in the present study, the association between the severity of ID and VPA-induced weight gain remains unknown. Further investigations are thus needed to confirm and further clarify the association between ID and VPAinduced weight gain.
A

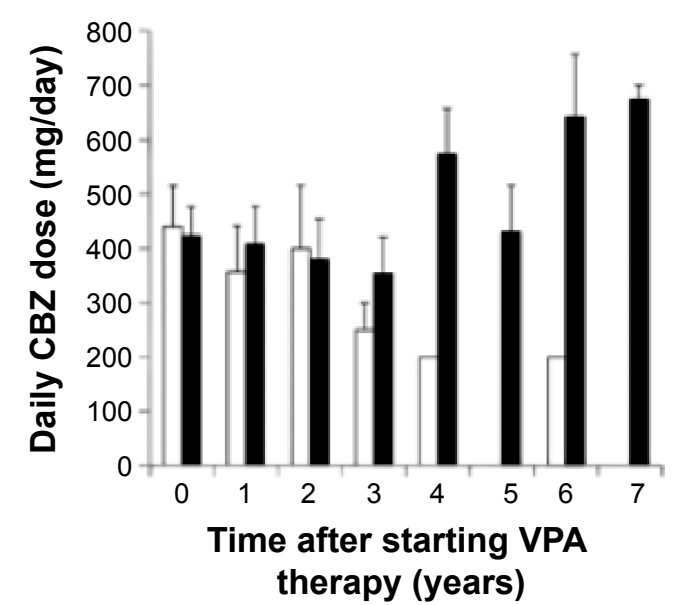

B

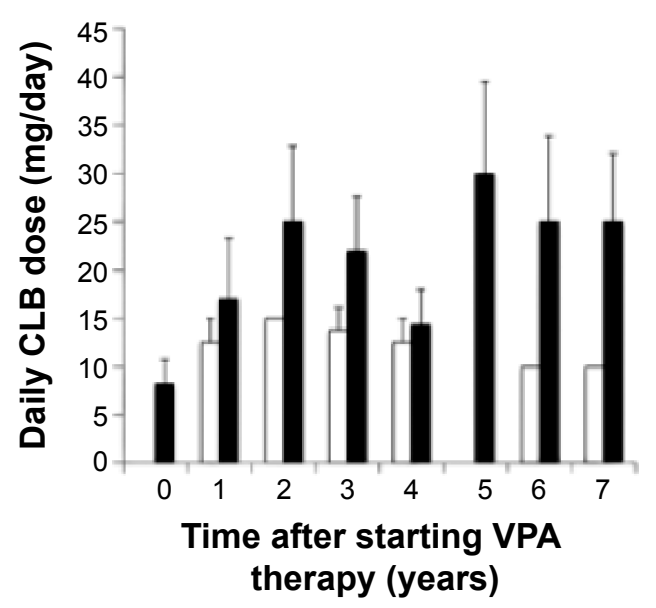

Figure 2 Daily doses of CBZ (A) or CLB (B) in patients treated with the co-administration of these antiepileptic drugs at each time point used in the evaluation of the longitudinal changes in the BMI gap during VPA therapy.

Note: The mean values are shown as solid and open bars in the patients with moderate ID and those without, respectively, and the SEs are shown as antennae. Abbreviations: CBZ, carbamazepine; CLB, clobazam; BMI, body mass index; VPA, valproic acid; ID, intellectual disability; SE, standard error. 


\section{Conclusion}

In conclusion, this is, to the best of our knowledge, the first study to show that patients with moderate ID may have a high risk of becoming overweight during VPA therapy. Additionally, this study demonstrated that the co-administration of carbamazepine and clobazam may be associated with VPA-induced weight gain in patients with moderate ID. The findings of the present study suggest that the potential for weight gain should be carefully monitored in VPA-treated patients with moderate ID, especially those receiving other co-administered antiepileptic drugs that facilitate weight gain, such as carbamazepine and clobazam.

\section{Acknowledgments}

The authors wish to thank all the study participants. This work was supported by grants-in-aid (Nos 25860117, 26360049) for scientific research from the Japanese Ministry of Education, Science, Sports and Culture.

\section{Author contributions}

All authors met the following criteria: (1) Substantial contributions to conception and design, acquisition of data, or analysis and interpretation of data; (2) drafting the article or revising it critically for important intellectual content; (3) final approval of the version to be submitted; and (4) agreement to be accountable for all aspects of the work in ensuring that questions related to the accuracy or integrity of any part of the work are appropriately investigated and resolved.

\section{Disclosure}

Norio Yasui-Furukori has received grant/research support or honoraria from and spoken for Asteras, Dainippon, Eli Lilly, GlaxoSmithKline, Janssen-Pharma, Meiji, Mochida, Merck Sharp and Dohme, Otsuka, Pfizer, Takada, and Yoshitomi. The remaining authors have declared that no competing interests exist.

\section{References}

1. Melville CA, Hamilton S, Hankey CR, Miller S, Boyle S. The prevalence and determinants of obesity in adults with intellectual disabilities. Obes Rev. 2007;8(3):223-230.

2. Maiano C. Prevalence and risk factors of overweight and obesity among children and adolescents with intellectual disabilities. Obes Rev. 2011;12(3):189-197.

3. Stancliffe RJ, Lakin KC, Larson S, et al. Overweight and obesity among adults with intellectual disabilities who use intellectual disability/ developmental disability services in 20 U.S. States. Am J Intellect Dev Disabil. 2011;116(6):401-418.

4. Begarie J, Maiano C, Leconte P, Ninot G. The prevalence and determinants of overweight and obesity among French youths and adults with intellectual disabilities attending special education schools. Res Dev Disabil. 2013;34(5):1417-1425.
5. Slevin E, Truesdale-Kennedy M, McConkey R, Livingstone B, Fleming P. Obesity and overweight in intellectual and non-intellectually disabled children. J Intellect Disabil Res. 2014;58(3):211-220.

6. Berg AT, Langfitt JT, Testa FM, et al. Global cognitive function in children with epilepsy: a community-based study. Epilepsia. 2008;49(4):608-614.

7. Rantanen K, Eriksson K, Nieminen P. Cognitive impairment in preschool children with epilepsy. Epilepsia. 2011;52(8):1499-1505.

8. Park J, Yum MS, Choi HW, Kim EH, Kim HW, Ko TS. Determinants of intelligence in childhood-onset epilepsy: a single-center study. Epilepsy Behav. 2013;29(1):166-171.

9. Reilly C, Atkinson P, Das KB, et al. Neurobehavioral comorbidities in children with active epilepsy: a population-based study. Pediatrics. 2014;133(6):e1586-e1593.

10. Nanau RM, Neuman MG. Adverse drug reactions induced by valproic acid. Clin Biochem. 2013;46(15):1323-1338.

11. Verrotti A, D’Egidio C, Mohn A, Coppola G, Chiarelli F. Weight gain following treatment with valproic acid: pathogenetic mechanisms and clinical implications. Obes Rev. 2011;12(5):e32-e43.

12. Belcastro V, D'Egidio C, Striano P, Verrotti A. Metabolic and endocrine effects of valproic acid chronic treatment. Epilepsy Res. 2013; 107(1-2):1-8.

13. Rauchenzauner M, Laimer M, Wiedmann M, et al. The novel insulin resistance parameters RBP4 and GLP-1 in patients treated with valproic acid: just a sidestep? Epilepsy Res. 2013;104(3):285-288.

14. Berg AT, Berkovic SF, Brodie MJ, et al. Revised terminology and concepts for organization of seizures and epilepsies: report of the ILAE Commission on Classification and Terminology, 2005-2009. Epilepsia. 2010;51(4):676-685.

15. Cole TJ, Bellizzi MC, Flegal KM, Dietz WH. Establishing a standard definition for child overweight and obesity worldwide: international survey. BMJ. 2000;320(7244):1240-1243.

16. Ben-Menachem E. Weight issues for people with epilepsy - a review. Epilepsia. 2007;48(Suppl 9):42-45.

17. Verrotti A, Manco R, Agostinelli S, Coppola G, Chiarelli F. The metabolic syndrome in overweight epileptic patients treated with valproic acid. Epilepsia. 2010;51(2):268-273.

18. Lakhanpal D, Kaur G. Valproic acid alters GnRH-GABA interactions in cycling female rats. Cell Mol Neurobiol. 2007;27(8):1069-1083.

19. Egger J, Brett EM. Effects of sodium valproate in 100 children with special reference to weight. BMJ. 1981;283(6291):577-581.

20. Zeng K, Wang X, Xi Z, Yan Y. Adverse effects of carbamazepine, phenytoin, valproate and lamotrigine monotherapy in epileptic adult Chinese patients. Clin Neurol Neurosurg. 2010;112(4):291-295.

21. Gaspari CN, Guerreiro CA. Modification in body weight associated with antiepileptic drugs. Arq Neuropsiquiatr. 2010;68(2):277-281.

22. Arya R, Anand V, Garg SK, Michael BD. Clobazam monotherapy for partial-onset or generalized-onset seizures. Cochrane Database Syst Rev. 2014;10:CD009258.

23. Alvarez N, Besag F, Iivanainen M. Use of antiepileptic drugs in the treatment of epilepsy in people with intellectual disability. J Intellect Disabil Res. 1998;42(suppl 1):1-15.

24. Raspall-Chaure M, Neville BG, Scott RC. The medical management of the epilepsies in children: conceptual and practical considerations. Lancet Neurol. 2008;7(1):57-69.

25. Wassenaar M, Leijten FS, Egberts TC, Moons KG, Uij1 SG. Prognostic factors for medically intractable epilepsy: a systematic review. Epilepsy Res. 2013;106(3):301-310.

26. Guerrini R, Rosati A, Segieth J, Pellacani S, Bradshaw K, Giorgi L. A randomized phase III trial of adjunctive zonisamide in pediatric patients with partial epilepsy. Epilepsia. 2013;54(8):1473-1480.

27. Cross JH, Auvin S, Patten A, Giorgi L. Safety and tolerability of zonisamide in paediatric patients with epilepsy. Eur J Paediatr Neurol. 2014;18(6):747-758.

28. Depner CM, Stothard ER, Wright KP Jr. Metabolic consequences of sleep and circadian disorders. Curr Diab Rep. 2014;14(7):507.

29. Vaessen TJ, Overeem S, Sitskoorn MM. Cognitive complaints in obstructive sleep apnea. Sleep Med Rev. 2015;19:51-58. 


\section{Publish your work in this journal}

Neuropsychiatric Disease and Treatment is an international, peerreviewed journal of clinical therapeutics and pharmacology focusing on concise rapid reporting of clinical or pre-clinical studies on a range of neuropsychiatric and neurological disorders. This journal is indexed on PubMed Central, the 'PsycINFO' database and CAS,

and is the official journal of The International Neuropsychiatric Association (INA). The manuscript management system is completely online and includes a very quick and fair peer-review system, which is all easy to use. Visit http://www.dovepress.com/testimonials.php to read real quotes from published authors.

Submit your manuscript here: http://www.dovepress.com/neuropsychiatric-disease-and-treatment-journal 